\title{
Supporting information for 'Selecting Carbon Nanotubes with Diameters of Less than 1 nm by Laser Pulses: An Ab Initio Exploration'
}

\author{
Yoshiyuki Miyamoto \\ Research Center for Computational Design of Advanced Functional Materials, \\ National Institute of Advanced Industrial Science and Technology (AIST), \\ Central 2, 1-1-1 Umezono, Tsukuba, 305-8568, Japan
}

\section{SI. Calculation of density of states obtained by the time-dependent density functional simulations}

The density of states (DOS) obtained by density functional theory (DFT) is approximated by computing the following equation

$$
\operatorname{DOS}^{D F T}(E)=\frac{1}{\pi} \operatorname{Im} \Sigma_{n, k} \frac{1}{E-E_{n, k}+i \delta},
$$

using the sampling $k$ points used in the current TDDFTMD simulations, five for $(8,0)(14,0)$ CNT array, seven for $(5,5)(8,8) \mathrm{CNT}$ array. In equation $(\mathrm{S} 1), E_{n, k}$ means $n$ th eigenvalues of the Kohn-Sham orbital with $k$ vector, and $\delta$ was set as $0.1 \mathrm{eV}$. On the other hand, the DOS obtained by TDDFT-MD is derived by

$$
\operatorname{DOS}^{T D D F T}(E)=\frac{1}{\pi} \operatorname{Im} \Sigma_{n, k} \frac{P^{T D D F T}(n, k)}{E-E_{n, k}+i \delta} .
$$

Here, the projection $P^{T D D F T}(n, k)$ is computed as

$$
P^{T D D F T}(n, k)=\Sigma_{m}\left|<\psi_{m, k}\right| \psi_{n, k}^{D F T}>\left.\right|^{2},
$$

where $\psi_{m, k}$ denotes $m$-th occupied time-dependent Kohn-Sham orbital with $k$ vector, whilie $\psi_{n, k}^{D F T}$ denotes the $n$-th Kohn-Sham orbital with $k$ vector obtained by the DFT calculation.

\section{SII. Examining stability of graphene nano-ribbon under linearly polarized femtosecond laser}

For the graphene nano ribbon (GNR), the $x, y$, and $z$ axes were set as the sheet-normal axis, the ribbon axis, and the remaining axis that is parallel to the sheet but normal to the ribbon axis, respectively, as shown in Figs. S1 (a) and (b). The unit cell of the GNR contained 42 carbon atoms, with 21 bars (composed $\mathrm{C}-\mathrm{C}$ bonds) of the armchair paths in the $z$-axis direction along the width of the ribbon. Two different polarizations of the laser field were examined. When the polarization vector of the laser field was set along the $z$ axis, the unit cell had periods of 8.0, 4.3, and $36.6 \AA$ along the $x, y$, and $z$ axes, respectively. When the polarization vector was set along the $x$ axis, the unit cell had periods of 12.0, 4.3, and 30.6 $\AA$ along the $x, y$, and $z$ axes, respectively. Four irreducible $k$ points were taken along the $y$ axis to integrate the charge density from the Bloch wave functions.

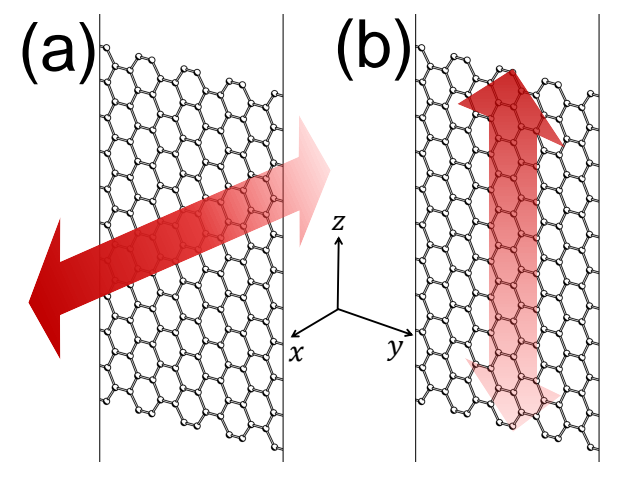

FIG. S1: (a) A periodic GNR with armchair-type edges. The period is in the $y$ direction. The red arrow indicates the polarization vector of the laser field, which is perpendicular to the GNR sheet ( $x$ direction). (b) Same as (a) but with the polarization vector parallel to the GNR sheet and perpendicular to the ribbon axis ( $z$ direction).

The behavior of a GNR sheet under pulsed laser irradiation with polarization parallel or perpendicular to the sheet was investigated. Figure S2 shows the results of first-principles simulations of the armchair-edged graphene sheets under pulsed laser irradiation with a fullwidth at half-maximum (FWHM) of $10 \mathrm{fs}$, a wavelength of $800 \mathrm{~nm}$, and a maximum laser field of $4 \mathrm{~V} / \AA$. The laser pulse shape is depicted in the upper panels of Figs. S2(a) and (b), and the time evolution of the heights of the bars composed of $\mathrm{C}-\mathrm{C}$ bonds parallel to the ribbon axis ( $y$ axis of Fig. S1) is displayed in the lower panels of Figs. S2(a) and (b). The heights are plotted along the direction of the ribbon width ( $z$ axis of Fig. S1) relative to the center of the ribbon. As shown in Fig. S2(a), when the optical field was perpendicular to the ribbon sheet, as indicated by the arrow in Fig. S1(a), the heights of the bars did not change. In contrast, as shown in Fig. S2(b), when the optical field was parallel to the ribbon sheet, as indicated by the arrow in Fig. S1(b), the bars near the top (bottom) edge of the GNR became higher (lower), indicating the exfoliation of the GNR edges during the repeated laser pulses. 
(a)

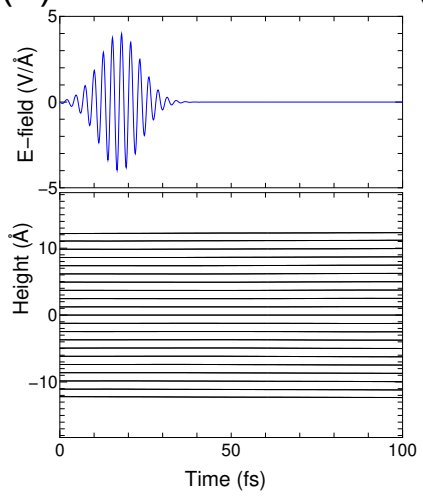

(b)

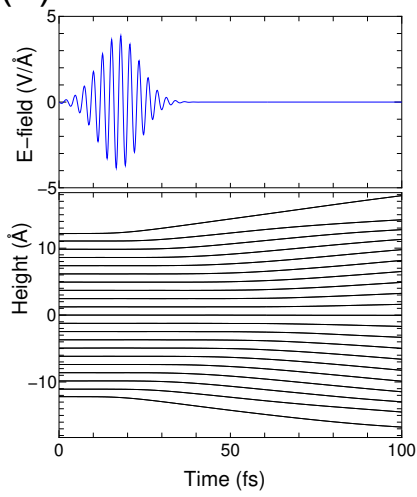

FIG. S2: TDDFT-MD simulations of an armchair GNR under pulsed laser irradiation with an FWHM of $10 \mathrm{fs}$, a wavelength of $800 \mathrm{~nm}$, and a maximum field intensity of $4 \mathrm{~V} / \AA$, where the polarization vector of the laser field was (a) perpendicular to the GNR sheet (as seen in Fig. S1(a)) and (b) parallel to the GNR sheet and perpendicular to the ribbon axis (as seen in Fig. S1(b)). The top panels depict the time evolution of the laser field intensity and the bottom panels show the time evolution of the heights of bars composed of the $\mathrm{C}-\mathrm{C}$ bonds in armchair paths parallel to the ribbon axis. The heights are plotted along the direction of the ribbon width ( $z$ axis of Fig. $\mathrm{S} 1)$ relative to the center of the ribbon.

\section{SIII. Analysis of survived narrower CNTs}

The influence of the excitation on survived CNT was analyzed by monitoring the kinetic energy of carbon atoms and electronic excitation after laser-selection of thinner CNTs, $(8,0)$ CNT in mixture with $(14,0)$ CNTs, and $(5,5)$ CNT in mixture with $(8,8)$ CNTs. Figure S3 (a) shows time variation of an averaged kinetic energies of carbon atoms of CNTs throughout the TDDFT-MD simulation shown in Figs. 2 (a) and (b), in main text. Meantime, Fig. S3 (b) shows the density of state of the time-dependent Kohn-Sham orbitals obtained by taking their projections on the static Kohn-Sham orbital of selected (survived) thinner CNTs.

According to the kinetic energies of ions shown in Fig. S3 (a), the lattice temperature of survived thinner CNT was estimated as around $2000 \mathrm{~K}$ by employing the equation $\frac{3}{2} K_{\mathrm{B}} T=E_{k i n}$, where $T$ is temperature, $E_{k i n}$ is ion's kinetic energy, and $K_{\mathrm{B}}$ is the Boltzmann's constant.

Although this lattice temperature is below the melting point of graphitic materials $(\sim 3000 \mathrm{~K})$, damaging of remained CNTs is very likely without thermal dissipation through inert gas in atmosphere or through substrates attached to CNTs.

Figure S3 (b) shows electronic excitation in remaining CNTs which was taken by projection of the timedependent Kohn-Sham orbital onto the Kohn-Sham orbital of the ground state of the survived CNT with the same atomic coordinates at the snapshot of the final moment of the TDDFT-MD simulation as displayed in Figs. 2 (a) and (b) in the main text. (Here the equations (S1)(S3) were applied, again) The Fig. S3 (b) indicates electronic state remains as excited thus relaxation of electron should occur in longer time-scale. It is very likely that the lattice temperature of the survived CNTs increases more unless radiative decay of excitation takes place. (a)

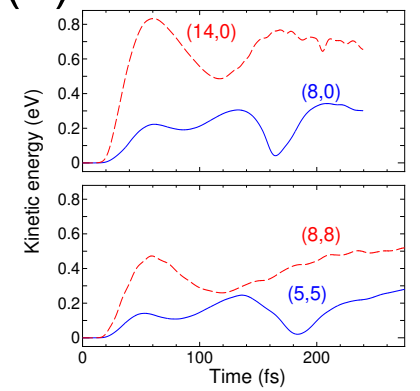

(b)

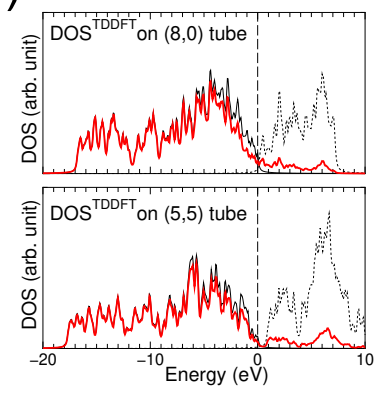

FIG. S3: (a) Time evolution of averaged kinetic energies of atoms in (upper) $(14,0)$ and $(8,0)$ CNTs and in (lower) $(8,8)$ and $(5,5)$ CNTs, throughout the TDDFT-MD simulation shown in Fig. 2 (a) and (b) of the main text. Solid (blue) and dashed (red) cures respectively denote the averaged kinetic energies of thiner and wider CNTs. (b) Thick (red) line represents density of states (DOS) for the time-dependent Kohn-Sham orbitals at the final snapshots of TDDFT-MD simulation as shown in Figs. 2 (a) and (b) in main text. The upper is for $(8,0)$ and $(14,0)$ CNTs, while the lower is for (lower) $(5,5)$ and $(8,8)$ CNTs. Each DOS curve was obtained by projection of the time-dependent Kohn-Sham orbitals onto the ground state Kohn-Sham orbitals of (upper) $(8,0)$ and of (lower) $(5,5)$ CNTs. Solid and dotted lines respectively show DOS of valence and conduction bands obtained by DFT ground state calculations. 